Letter

\title{
Design of a Miniaturized Rectangular Multiturn Loop Antenna for Shielding Effectiveness Measurement
}

\author{
Sangwoon Youn ${ }^{1}$, Tae Heung Lim ${ }^{1}$, Eunjung Kang ${ }^{1}$, Dae Heon Lee ${ }^{2}$, Ki Baek Kim ${ }^{2}$ and \\ Hosung Choo ${ }^{1, *(1)}$ \\ 1 School of Electronic and Electrical Engineering, Hongik University, Seoul 04066, Korea; \\ tirano88@naver.com (S.Y.); qpzm_0105@naver.com (T.H.L.); lemon_ya@naver.com (E.K.) \\ 2 Affiliated Institute of Electronics and Telecommunications Research Institute, Daejeon 30147, Korea; \\ leedh@nsr.re.kr (D.H.L.); kbkim11@nsr.re.kr (K.B.K.) \\ * Correspondence: hschoo@hongik.ac.kr
}

Received: 25 April 2020; Accepted: 2 June 2020; Published: 3 June 2020

\begin{abstract}
This paper proposes a novel miniaturized rectangular loop antenna sensor consisting of a multiturn wire and a cuboid ferrite core. The lateral surface of the ferrite core is tightly wound by the multiturn wire. To verify its feasibility, the antenna sensor is fabricated, and the antenna factor (AF) levels are measured using the three-antenna method from the very low frequency (VLF) to the high-frequency (HF) bands. The measured AF levels are $31.8 \mathrm{~dB}$ (with a covering plastic case) and $33.1 \mathrm{~dB}$ (without a covering plastic case) at $30 \mathrm{kHz}$. In addition, the proposed antenna is employed in the shielding effectiveness measurement of a small commercial cabinet to observe its suitability for shielding effectiveness (SE) measurement of small shielding enclosures. The SE values averaged over the frequency range from $10 \mathrm{kHz}$ to $3 \mathrm{MHz}$ are $4.1 \mathrm{~dB}$ and $12 \mathrm{~dB}$ in the horizontal and vertical polarizations, respectively.
\end{abstract}

Keywords: VLF antenna miniaturization; ferrite loop antenna; multiturn loop antenna; AF enhancement; SE measurement

\section{Introduction}

In recent electronic warfare, shielding facilities have become essential to protect important electrical devices from electromagnetic pulse (EMP) attacks [1-3]. For low-cost maintenance, small shielding enclosures that can only shield electrical devices have been gradually demanded to protect these devices more efficiently [4-7]. Shielding effectiveness (SE) for such small shielding enclosures also needs to be measured at various frequency ranges to determine the EMP strength that the enclosures can endure. In conventional SE measurements based on IEEE standard 299.1, loop antennas are generally used to measure SE from the very-low-frequency (VLF) band to the high-frequency (HF) band in a large shielding room [8-12]. However, SE measurements of small shielding enclosures are significantly problematic using conventional SE measurement systems due to the large size of the loop antenna [13]. For example, the small shielding enclosure usually contains one side of the enclosure that is less than $700 \mathrm{~mm}$ according to the IEEE standard 299. 1. However, it is difficult to accurately measure the SE levels at edges or sides of the small shielding enclosure, because the physical size of the conventional loop antenna sensor, i.e., AH-systems [14], has a large diameter of $300 \mathrm{~mm}$. To overcome this issue, research on miniaturization techniques for loop antennas has been extensively conducted by employing various loop designs [15-18], printed loops with ground planes [19], and adding lumped elements [20-22]. Although these approaches can miniaturize the physical size of loop antennas, such techniques usually require sophisticated and expansive fabrication processes. In addition, these loop antennas often have high antenna factors (AFs) in the VLF band, 
which can degrade the accuracy of the SE measurement. Thus, to improve the accuracy of the SE measurement, various antenna sensor structures have been introduced [23,24], such as shielded loop antennas [25], two-port loop antennas [26], loop antennas with parasitic elements [27], and Moebius loop antennas [28]. These previous studies have been successful in improving measurement accuracy with antenna sensors operating in a narrow frequency band. However, the SE measurement using such narrow band antenna sensors requires a lot of manpower and time, because the antenna sensors need to be replaced depending on the operating frequency bands. In addition, there is still a need for in-depth research on the miniaturization of the loop antenna with broadband characteristics in the VLF band, which can maintain low AF levels.

In this paper, we propose a novel design structure of a compact rectangular loop antenna sensor composed of a multiturn wire and a cuboid ferrite core. The proposed antenna has a cuboid-shaped ferrite core wound multiple times by a thick wire to concentrate the magnetic flux density, which can reduce the AF level in the VLF band. Then, the antenna characteristics such as the impedance and the $\mathrm{AF}$ in terms of the frequency are estimated using the FEKO EM simulator [29]. The theoretical AF is also derived by the multiturn loop formula and compared to the simulation results. To verify the feasibility, the proposed antenna sensor is fabricated to measure its AF characteristics using the three-antenna method from the VLF to HF bands. The variations in the AFs according to the frequency are observed depending on the important antenna geometry parameters. The antenna sensor is finally utilized to carry out the SE measurement of the commercial small metallic cabinet, and the results demonstrate that it is suitable for measuring the SE of small shielding enclosures from VLF to HF bands.

\section{Proposed Antenna and Theory}

\subsection{Theoretical Background}

Figure 1a illustrates a conceptual geometry of the rectangular multiturn loop antenna in a receiving mode with a side length of $a$ and $N$ turns. When an electromagnetic planewave propagates toward the loop antenna at the incident angle of $\theta$, an open-circuit voltage is then induced across nodes 1 and 2. To easily understand this concept, the receiving loop antenna can be described by a Thevenin equivalent circuit as shown in Figure $1 b$, and the circuit components are composed of an input impedance of $Z_{i n}$, a load impedance $Z_{L}$ of $50 \Omega$, and an open-circuit voltage of $V_{o c}$. As written in Equation (1), the open-circuit voltage $V_{o c}$ can be expressed by an area of the physical loop antenna size and the amplitude of the magnetic field intensity [30,31]. The load voltage $V_{L}$ can be calculated by the voltage ratio of the input and load impedances as expressed in Equation (2). Then, the AF of the loop antenna is derived by the ratio between the magnetic field intensity and the loaded voltage as noted in Equation (3) [32].

$$
\begin{gathered}
V_{o c}=j \omega a^{2} \mu_{0} H N \sin \theta, \\
V_{L}=V_{o c} \frac{Z_{L}}{Z_{i n}+Z_{L}}, \\
\mathrm{AF}=20 \log _{10}\left(H / V_{L}\right) .
\end{gathered}
$$

To reconfirm the theoretical result, we model the multiturn loop antenna using the FEKO EM simulator based on the method of moments $(\mathrm{MoM})$ with piecewise tetrahedron meshes of $1 / 1000$ wavelength at $20 \mathrm{MHz}$. The antenna has a rectangular loop structure with a side length of $a$ and $N=5$. The simulation model was analyzed with piecewise tetrahedron meshes of $1 / 1000$ wavelength for $20 \mathrm{MHz}$. We also applied double precision option and low-frequency stabilization to obtain the suitable numerical simulation results. Figure 2 presents AF comparisons of the theoretical result and simulation according to frequency. The theoretical result is derived by Equations (1)-(3) using the input impedance of the loop antenna, which is indicated by the solid line. The dashed line shows the simulation result obtained using the magnetic field intensity and loaded voltage under the same conditions as in theory. AFs by the theoretical and simulation results show a similar trend in most of 
frequencies, with values of $38.8 \mathrm{~dB}$ and $38.6 \mathrm{~dB}$ at $30 \mathrm{kHz}$, respectively. However, the AF levels for both results have a slight difference in the high-frequency band because the loop antenna model in the equation makes it difficult to consider the detailed antenna parameters.

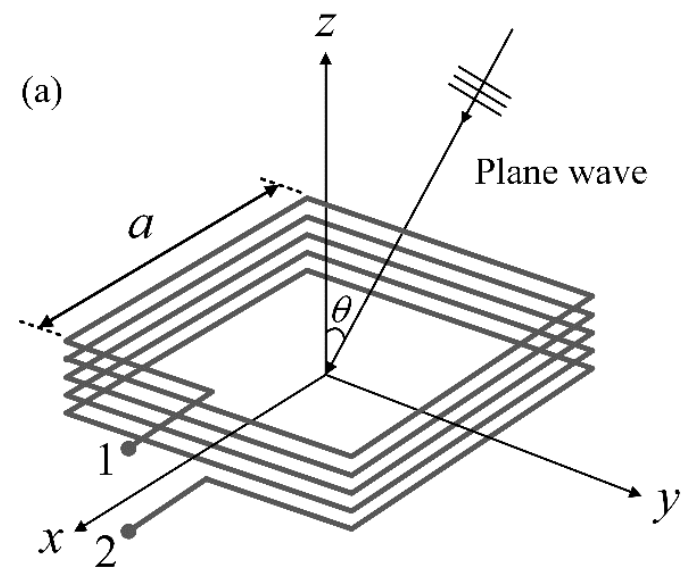

(b)

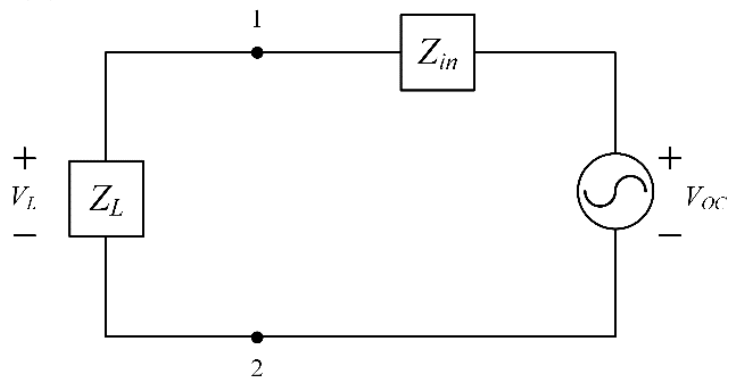

Figure 1. Conceptual figure of the rectangular multiturn loop antenna: (a) planewave toward the rectangular loop antenna; (b) thevenin equivalent circuit model of the loop antenna.

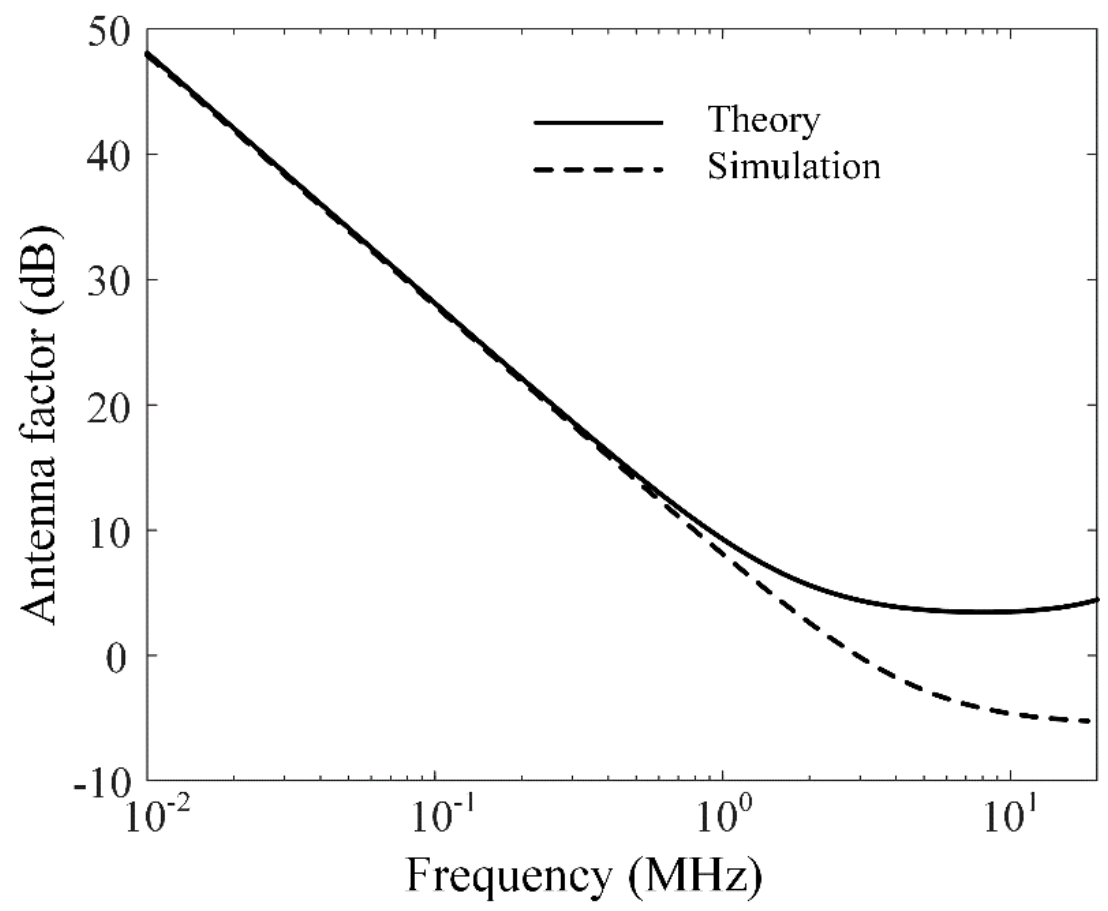

Figure 2. Antenna factor (AF) comparisons of the theoretical result and simulation.

\subsection{Proposed Antenna Structure and Simulation}

Figure $3 \mathrm{a}, \mathrm{b}$ show the geometry of the rectangular multiturn loop antenna sensor. The proposed antenna consists of a rectangular multiturn loop and a cuboid-shaped ferrite core $\left(\mu_{r}=210\right)$. The cuboid-shaped ferrite core, having a thickness of $h$ with a width of $w$, is wound by a thick wire with a diameter $t$, which can miniaturize the antenna's physical structure size with a low AF in the VLF band. In addition, the $N$ turns of the rectangular loop wire helps to concentrate a strong magnetic flux density in the center of the antenna. The detailed design parameters are listed in Table 1. Figure $4 \mathrm{a}-\mathrm{d}$ show the AF results in accordance with the variations of the important geometry parameters $(h, N, t$, and $w)$. As can be seen in Figure $4 \mathrm{a}$, the $\mathrm{AF}$ levels in the entire frequency range 
decrease due to the ferrite property that focuses the magnetic flux density in the center of the antenna when the ferrite thickness $h$ increases. Figure $4 \mathrm{~b}$ presents that the AF levels dramatically decrease at low frequencies, but $\mathrm{AF}$ tends to increase at high frequencies, when the number of the wire turns increases. Figure $4 \mathrm{c}$ illustrates the AF levels in accordance with the variation of the wire diameter $t$. The AF levels in the HF bands are reduced as $t$ increases, because the low input impedance characteristic at the high frequency can induce the high load voltage as indicated in Figure 1b. The low AF levels are obtained as the ferrite core width $w$ increases, as shown in Figure $4 \mathrm{~d}$. Note that the magnetic flux passing through the center of the ferrite core becomes dense and can lead to the high induced open-circuit voltage. The resulting parametric study demonstrates that the proposed antenna sensor can be miniaturized by the ferrite core with the multiturn thick wire.

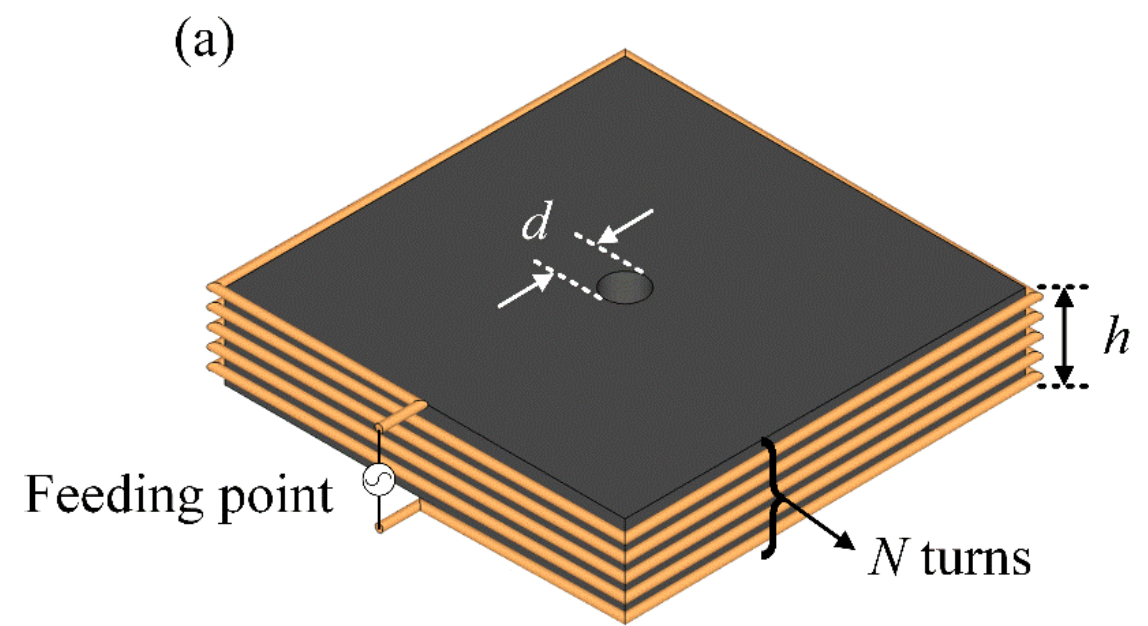

(b)

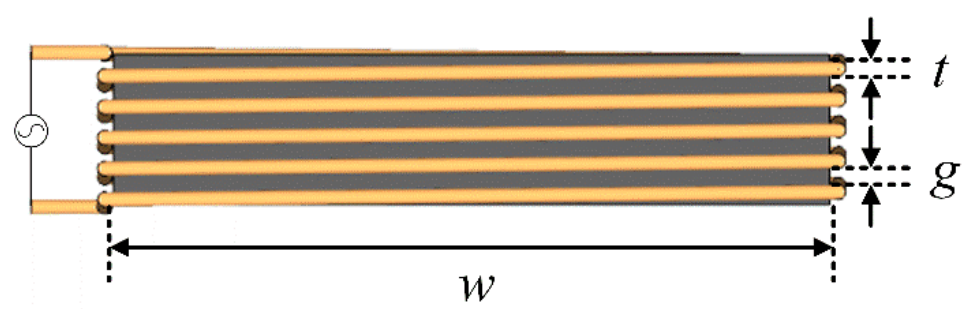

Figure 3. Geometry of the proposed antenna: (a) isometric view; (b) side view.

Table 1. Design parameters of the proposed antenna.

\begin{tabular}{cc}
\hline Parameters & Values \\
\hline$t$ & $1 \mathrm{~mm}$ \\
$g$ & $4 \mathrm{~mm}$ \\
$h$ & $21 \mathrm{~mm}$ \\
$w$ & $100 \mathrm{~mm}$ \\
$d$ & $10 \mathrm{~mm}$ \\
$N$ & 5 turns \\
\hline
\end{tabular}




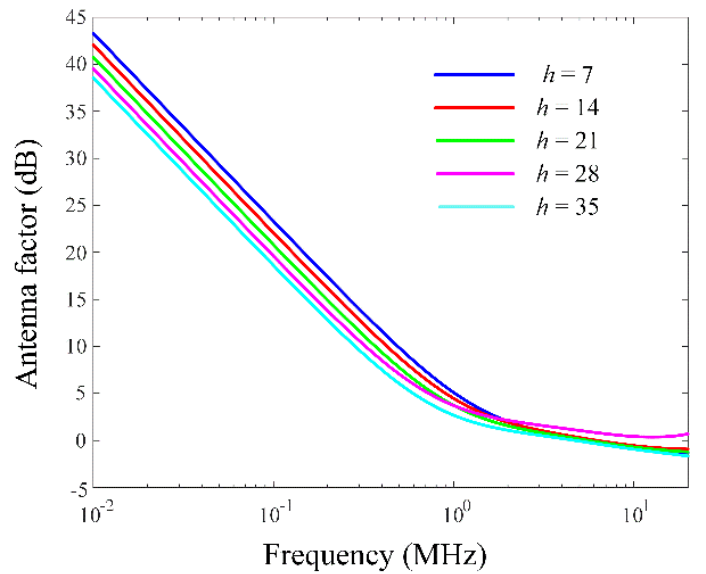

(a)

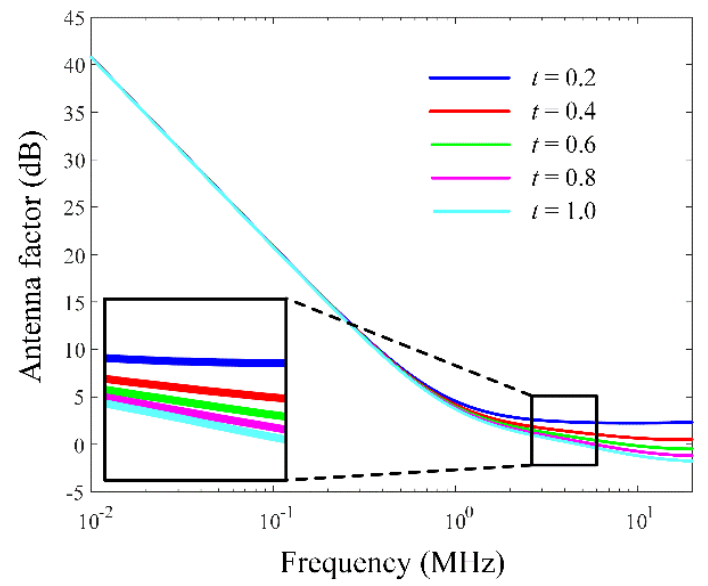

(c)

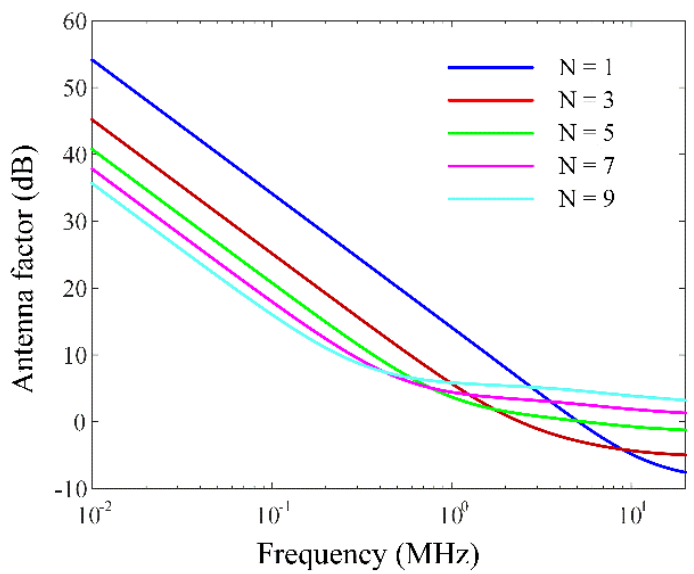

(b)

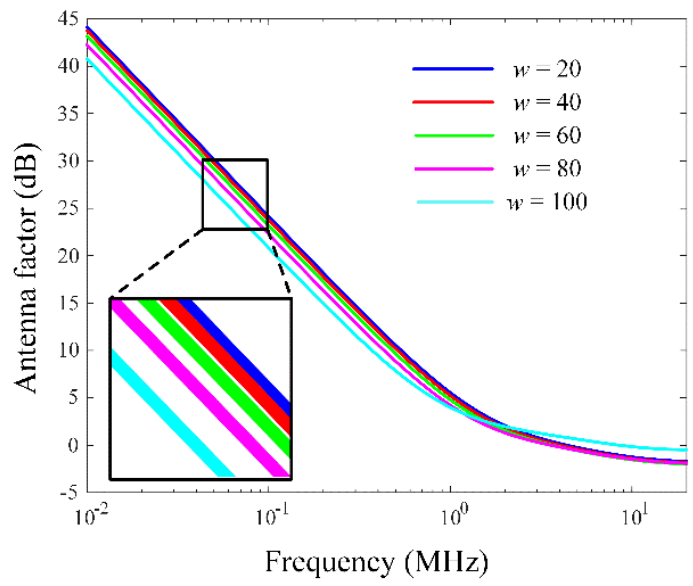

(d)

Figure 4. AF variation according to design parameters: (a) AF in accordance with the variation of $h$, (b) AF in accordance with the variation of $N$, (c) AF in accordance with the variation of $t$, and (d) AF in accordance with the variation of $w$.

\section{Antenna Fabrication and Measured Results}

Figure $5 \mathrm{a}-\mathrm{c}$ present photographs of the fabricated antenna sensor, a covering plastic case, and an assembled structure. The multiturn loop antenna has a simple structure, which is composed of the copper wire coated by polyethylene and the ferrite core (SD $100 \times 100 \times 7$, Samwha). The lateral surfaces of the cuboid-shaped ferrite are wound by the wire with five turns, which are firmly wrapped by Styrofoam to protect the antenna from external shocks. Then, this fabricated antenna is inserted in the covering plastic case being assembled with nuts to improve durability, and the ends of the multiturn loop are directly connected to the N-type connector as a feeder. The covering case makes it easy to handle and enables practical SE measurements. To examine AF characteristics, the fabricated antenna is measured by applying the three-antenna method [33]. In this method, S-parameters are obtained by utilizing two reference antennas and one test antenna. For two reference antennas, we fabricate two single-turn circular wire loop antenna having a loop diameter of $30 \mathrm{~cm}$ with a SMA feeder. Figure 6 represents the measured and simulated AF levels of the proposed rectangular multiturn antenna. The measured AF values with and without the covering plastic case are $31.8 \mathrm{~dB}$ and $33.1 \mathrm{~dB}$ and agree with the simulation of $30.9 \mathrm{~dB}$ at $30 \mathrm{kHz}$. The slight difference of the AF levels between the simulations and measurements in high frequency occurs due to the fabrication error, insufficient material parameters, and simplified feeding structure. We also compare the antenna performances, such as operating frequency bands, antenna geometry dimensions, and AF levels at $30 \mathrm{kHz}$, between 
the proposed antenna sensor and the reference loop antennas. The detailed values are listed in Table 2. The results confirm that the proposed sensor can maintain low AF values from the VLF band to the HF band despite the small physical antenna size, which is downsized to $33 \%$ of the conventional loop antenna. We additionally carried out the SE measurement of a commercial metallic cabinet using the proposed antenna in order to confirm the feasibility. In IEEE standard 299.1, SE is defined as an indicator of the shielding performance for an enclosing structure, which can be obtained by comparing the field intensities with and without the shielding structure from an external electromagnetic source. Figure 7a-c show the SE measurement setup with measurement equipment such as a function generator, a spectrum analyzer, and a power amplifier. We use the function generator that is connected to the proposed antenna and the power amplifier with a gain of $18 \mathrm{~dB}$ for the transmitting part. For the receiving part, the identical antenna is connected to the spectrum analyzer to measure the field strength. Figure 8 shows the result of the SE measurement of the metallic cabinet by the vertical and horizontal polarization, respectively. The cabinet has average SE values of $12 \mathrm{~dB}$ for a vertical polarization and $4.1 \mathrm{~dB}$ for a horizontal polarization.

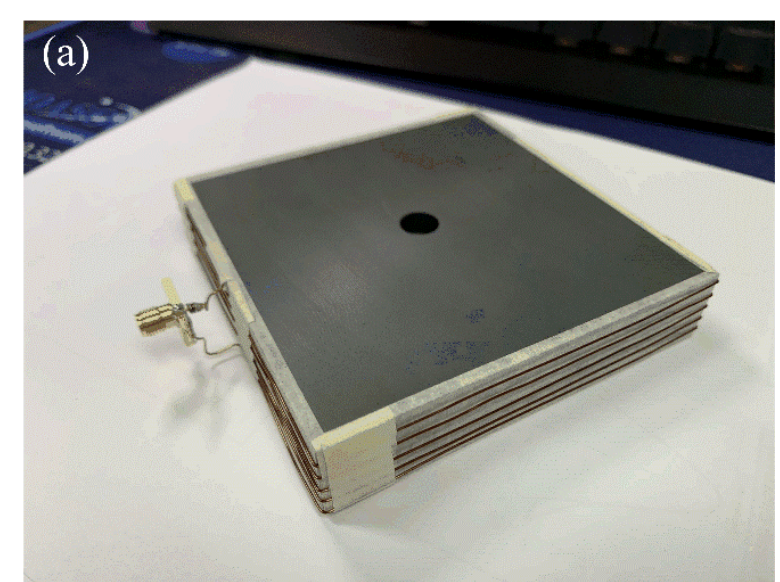

(b)

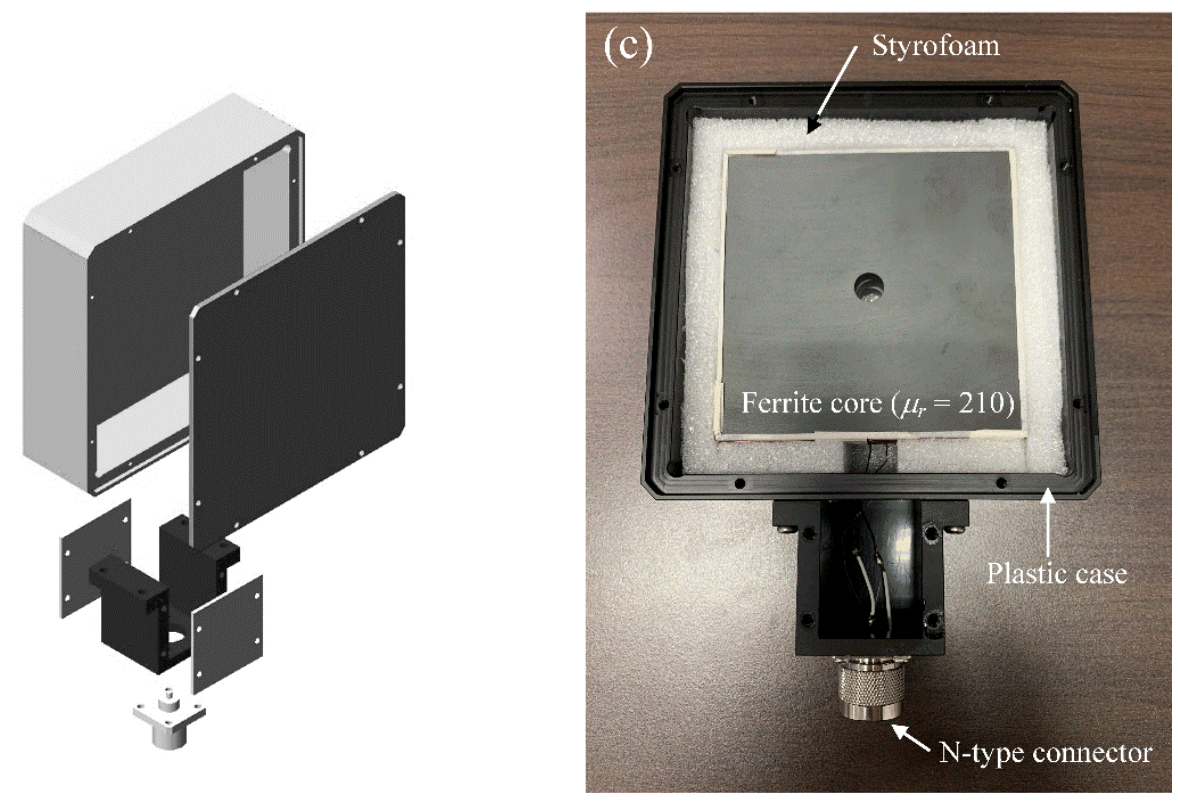

Figure 5. Photographs of the fabricated and assembled antenna: (a) isometric view of the proposed antenna, (b) casing parts, and (c) assembled antenna. 


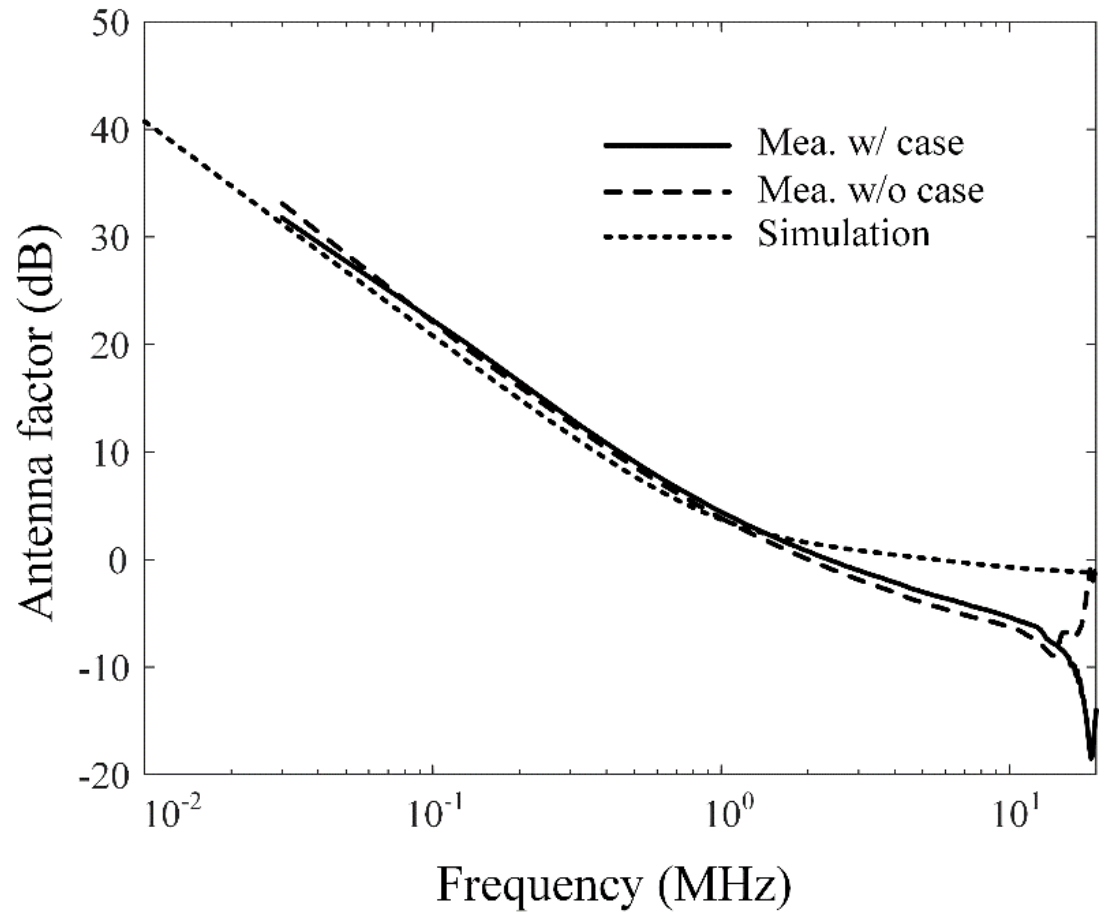

Figure 6. AF comparisons of the simulation and measurements.
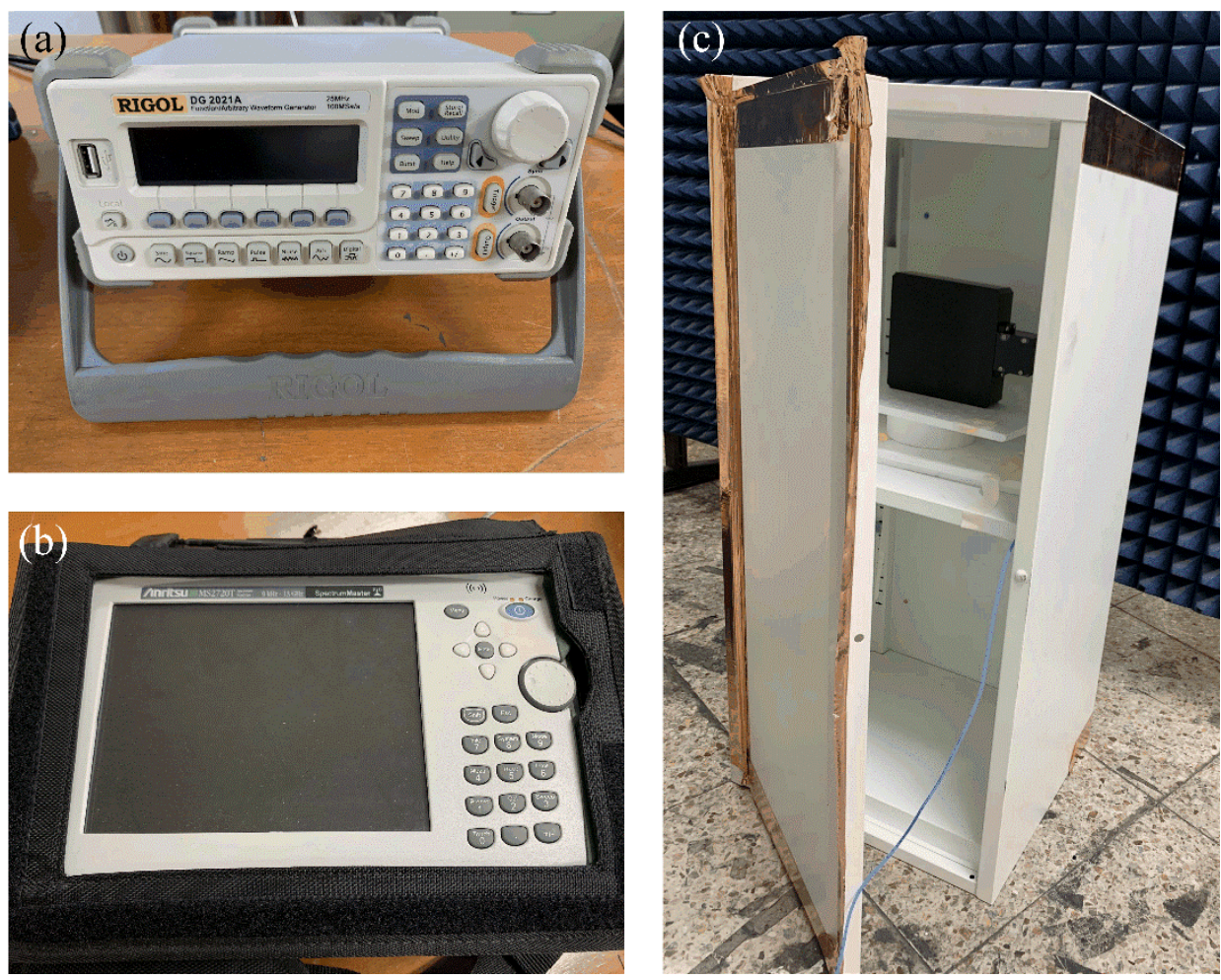

Figure 7. SE measurement setup: (a) function generator; (b) spectrum analyzer; and (c) loop antenna within the commercial metallic cabinet. 
Table 2. Antenna performance comparison.

\begin{tabular}{cccc}
\hline Antenna & Frequency & Dimension (mm) & AF (@30 kHz) \\
\hline$[10]$ & $9 \mathrm{kHz}-10 \mathrm{MHz}$ & $60 \times 60 \times 6$ & - \\
[30] & $1 \mathrm{MHz}-30 \mathrm{MHz}$ & $152 \times 152 \times 3.7$ & - \\
A.H. system & $10 \mathrm{kHz}-30 \mathrm{MHz}$ & $300 \times 300$ & $28 \mathrm{~dB}$ \\
$\begin{array}{c}\text { (SAS-563P) [14] } \\
\text { ETS-lindgren }\end{array}$ & $9 \mathrm{kHz}-30 \mathrm{MHz}$ & $600 \times 600 \times 38$ & $27 \mathrm{~dB}$ \\
$\quad$ (6512) [34] & $9 \mathrm{kHz}-30 \mathrm{MHz}$ & $600 \times 600$ & $21 \mathrm{~dB}$ \\
TDK (LP-0930P) [35] & $900 \times 100 \times 21$ & $31.8 \mathrm{~dB}$ \\
Proposed Ant. & $10 \mathrm{kHz}-30 \mathrm{MHz}$ & 100 \\
\hline
\end{tabular}

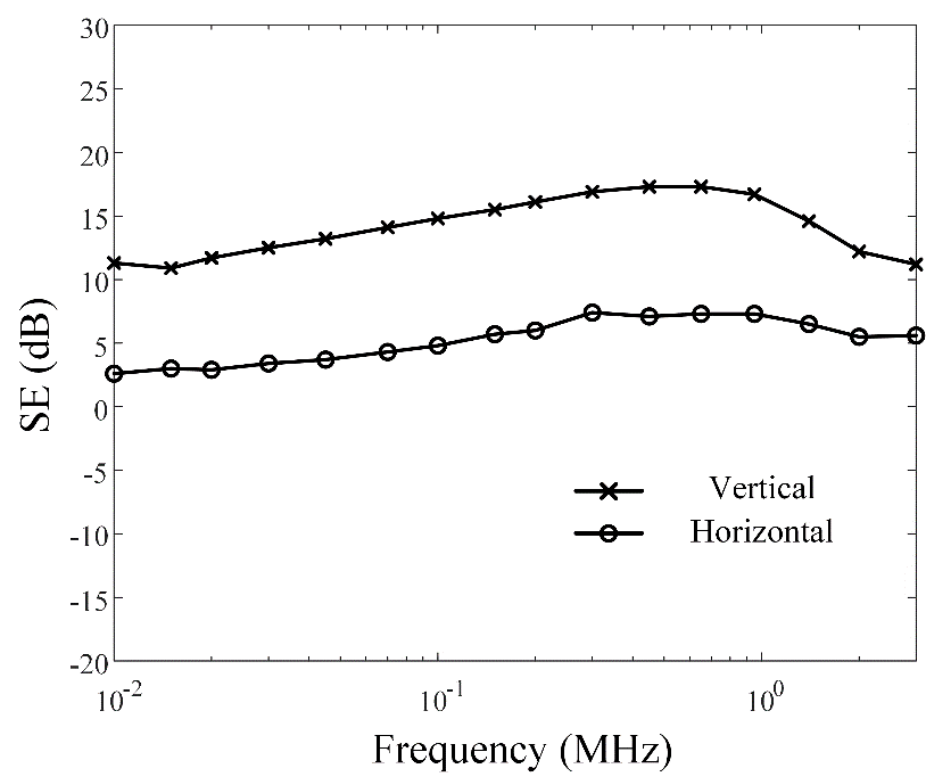

Figure 8. Measured SE in horizontal and vertical polarizations.

\section{Conclusions}

In this paper, we proposed a novel design structure of a compact rectangular loop antenna composed of a multiturn wire and a cuboid ferrite core. The antenna sensor had a cuboid-shaped ferrite core wound by a thick wire in order to concentrate the magnetic flux density in the center of the antenna, which could reduce the AF levels in the VLF band. The measured AF levels of the proposed antenna were $31.8 \mathrm{~dB}$ (with a covering plastic case) and $33.1 \mathrm{~dB}$ (without a covering plastic case) at $30 \mathrm{kHz}$, which agreed well with the simulation of $30.9 \mathrm{~dB}$. The SE measurement results of the commercial cabinet over the frequency range from $10 \mathrm{kHz}$ to $3 \mathrm{MHz}$ were $4.1 \mathrm{~dB}$ and $12 \mathrm{~dB}$ in the horizontal and vertical polarizations, respectively.

Author Contributions: Conceptualization, S.Y., T.H.L., D.H.L., K.B.K., and E.K.; methodology, S.Y. and T.H.L.; software, S.Y.; validation, S.Y., T.H.L., and E.K.; formal analysis, S.Y., T.H.L., D.H.L., and K.B.K.; investigation, S.Y., E.K., D.H.L., and K.B.K.; writing-original draft preparation, S.Y. and T.H.L.; writing-review and editing, S.Y. and H.C.; visualization, S.Y.; supervision, H.C.; project administration, H.C.; funding acquisition, H.C. All authors have read and agreed to the published version of the manuscript.

Funding: This research was funded by affiliated institute of ETRI.

Conflicts of Interest: The authors declare no conflict of interest. 


\section{References}

1. Marcello, D.A.; Maria, S.S. Theoretical and experimental characterization of the EMP-interaction with composite-metallic enclosures. IEEE Trans. Electromagn. Compat. 2000, 42, 152-163.

2. Qi, F.L.; Wen, Y.Y.; Ming, F.X.; Jun, F.M.; Liu, Q.H. Shielding characterization of metallic enclosures with multiple slots and a thin-wire antenna loaded: Multiple oblique EMP incidences with arbitrary polarizations. IEEE Trans. Electromagn. Compat. 2009, 51, 284-292.

3. Lim, N. Conductive concrete structure for EMP protection of critical infrastructure facilities. Lett. Electromagn. Compat. Pract. Appl. 2019, 1, 26-33.

4. Qingguo, W.; Erwei, C.; Zhaoming, Q. On the shielding effectiveness of small-dimension enclosures using a reverberation chamber. IEEE Trans. Electromagn. Compat. 2011, 53, 562-569.

5. Christopher, L.H.; David, A.H.; Marco, S.; John, M.L.; Jason, C.; Galen, K.; Andrew, C.M.; Yuhui, H. Use of reverberation chambers to determine the shielding effectiveness of physically small, electrically large enclosures and cavities. IEEE Trans. Electromagn. Compat. 2008, 50, 770-782.

6. Zhou, Z.; Gui, L.; Liu, D.; Lv, L.; Lu, D.; Lang, L. Simulation and measurement for shielding effectiveness of small size metal enclosure. IET Sci. Meas. Technol. 2017, 11, 25-29.

7. Ali, S.; Mojtaba, J. Shielding effectiveness measurement for extremely small dimension enclosures. IEEE Trans. Electromagn. Compat. 2019, 61, 1740-1745.

8. Sebastiano, D.F.; Mirko, M.; Bernardo, T.; Carmine, Z. Shielding effectiveness measurements for ferromagnetic shields. IEEE Trans. Instrum. Meas. 2009, 58, 115-121.

9. Yessica, A.; Andrew, H.; Oliver, C.L. Evaluation of near-field electromagnetic shielding effectiveness at low frequencies. IEEE Sens. J. 2019, 19, 121-128.

10. Amin, F.; Mohamed, B.; Fabrice, D.; Nabil, B.; Frederic, L.; Lionel, P. A new methodology to predict the magnetic shielding effectiveness of enclosures at low frequency in the near field. IEEE Trans. Magn. 2015, 51, 1-4.

11. Moser, J.R. Low-Frequency Low Impedance Electromagnetic Shielding. IEEE Trans. Electromagn. Compat. 1988, 30, 202-210. [CrossRef]

12. Han, H.L.; Jae, W.L.; Jong, H.K.; Jong, H.H.; Chang, H.H. Prediction of radio-wave propagation in a shield room: Measurement, simulation, and theoretical method. J. Electromagn. Eng. Sci. 2020, 20, 45-54.

13. Soyeon, K.; Hyunchul, S. An Ultra-Wideband Conformal Meandered Loop Antenna for Wireless Capsule Endoscopy. J. Electromagn. Eng. Sci. 2019, 19, 101-106.

14. A.H. Systems, Inc. SAS-563P. Available online: https://www.ahsystems.com/catalog/SAS-563B.php (accessed on 30 October 2019).

15. Zahid, Z.; Qu, L.; Kim, H.H.; Kim, H. Circularly polarized loop-type ground radiation antenna for IoT applications. J. Electromagn. Eng. Sci. 2019, 19, 153-158. [CrossRef]

16. Dohoon, K.; Yongjin, K. Small low-profile loop wideband antennas with unidirectional radiation characteristics. IEEE Trans. Antennas Propag. 2007, 55, 72-77.

17. Guangping, Z.; Glenn, S.S. The multiturn half-loop antenna. IEEE Trans. Antennas Propag. 1994, 42, $750-754$.

18. Lingnan, S.; Yahya, R.S. Miniaturized loop antennas for wireless brain-machine interfaces: Efficiency enhancement and link characterizations. In Proceedings of the Antennas and Propagation (APSURSI), 2016 IEEE International Symposium, Fajardo, Puerto Rico, 26 June-1 July 2016; pp. 879-880.

19. Kin, L.W.; Wei, Y.C.; Ting, W.K. On-board printed coupled-fed loop antenna in close proximity to the surrounding ground plane for penta-band WWAN mobile phone. IEEE Trans. Antennas Propag. 2011, 59, 751-757.

20. Liu, H.; Cheng, Y.; Yan, M. Electrically small loop antenna standing on compact ground in wireless sensor package. IEEE Antennas Wirel. Propag. Lett. 2011, 10, 438-441.

21. Pavel, T.; Irina, M.; Michail, D.; Orest, V.; Irina, V. Electrically small loop antennas for RFID applications. IEEE Antennas Wirel. Propag. Lett. 2015, 14, 1786-1789.

22. Gokhan, M.; Saurabh, G.; Kubilay, S.; John, L.V. Small wideband double-loop antennas using lumped inductors and coupling capacitors. IEEE Trans. Antennas Propag. 2011, 10, 107-110.

23. He, D. PT-level high-sensitivity magnetic sensor with amorphous wire. Sensors 2019, 20, 161. [CrossRef]

24. Spyridon, S.; Adyl, M.E.G.; Fabien, M.; Philippe, P. Fabrication and characterization of a flexible fluxgate sensor with pad-printed solenoid coils. Sensors 2020, 20, 2275. [CrossRef] 
25. Brian, B.T.; Anthony, G. Planar shielded-loop resonators. IEEE Trans. Antennas Propag. 2014, 62, 3310-3320.

26. Mani, K.; Kamal, S. An accurate circuit model for input impedance and radiation pattern of two-port loop antennas as E-and H-probe. IEEE Trans. Antennas Propag. 2017, 65, 114-120.

27. Rong, L.L.; Gerald, D.; Joy, L.; Manos, M.T. Investigation of circularly polarized loop antennas with a parasitic element for bandwidth enhancement. IEEE Trans. Antennas Propag. 2005, 53, 3930-3938.

28. Ofer, A. Moebius loop antenna system stability analysis under parameters variation. In Proceedings of the IEEE International Conference on Microwaves, Antennas, Communications and Electronic Systems, Tel-Aviv, Israel, 13-15 November 2017; pp. 1-5.

29. FEKO EM Simulation Software, Altair Engineering Inc. Available online: http://www.altair.co.kr (accessed on 30 September 2003).

30. Constantine, A.B. Antenna Theory Analysis and Design, 3rd ed.; Wiley-Interscience: Manhattan, NY, USA, 2005; pp. 245-246.

31. Boris, L. Field of a rectangular loop. IEEE Trans. Antennas Propag. 2004, 52, 948-952.

32. Masanori, I.; Koji, K. A simple method by measuring the impedance for evaluation of magnetic antenna factor of a loop antenna. IEICE Electron. Express 2006, 3, 92-96.

33. Masanori, I.; Koji, K. Impedance method for a shielded standard loop antenna. IEEE Trans. Instrum. Meas. 2007, 56, 422-425.

34. ETS-lindgren. 6512. Available online: http://www.ets-lindgren.com/datasheet/antennas/loops/4009/400905 (accessed on 28 April 2016).

35. TDK RF Solutions. LP-0930P. Available online: https://www.tdkrfsolutions.tdk.com/products/loop-antennas/ passive-loop-antenna-lp-0930p (accessed on 30 May 2016).

(C) 2020 by the authors. Licensee MDPI, Basel, Switzerland. This article is an open access article distributed under the terms and conditions of the Creative Commons Attribution (CC BY) license (http://creativecommons.org/licenses/by/4.0/). 